\title{
El asma bronquial. Consideraciones clínicas, ideológicas y terapéuticas
}

Miguel Costa

\section{INDICE}

\section{INTRODUCCION.}

1. Caracterización.

2. Aspectos etiológicos.

3. Patrones conductuales del paciente en el manejo de su enfermedad.

4. Aproximaciones terapéuticas.

4.1. Medidas generales.

4.2. Medicación.

4.3. Hiposensibilización.

4.4. Manejo comportamental.

4.4.1. Análisis conductual.

4.4.1.1. Estímulos antecedentes.

4.4.1.2. Estímulos concurrentes.

4.4.1.3. Estimulos consecuentes.

4.4.2. Objetivos.

4.4.2.1. Habilidades de observación y discriminación.

4.4.2.2. Habilidades en el control y reducción de la ansiedad.

4.4.2.3. Reducción de la sensibilidad.

4.4.2.4. Control de consecuencias reforzantes.

4.4.3. Técnicas y procedimientos terapéuticos. 


\section{INTRODUCCION}

En los últimos años se viene observando la importancia creciente de la práctica e investigación psicológica en un campo que hasta hace poco parecía privativo de la medicina. La publicación de artículos sobre terapia del comportamiento que tienen relación con aspectos psicofisiológicos no deja de ir en aumento (Ciminero, Doleys y Williams, 1978) y comienza a popularizarse el término «Medicina Conductual» (Schwartz, 1977) para describir el campo relacionado con el desarrollo de los conocimientos y técnicas de la ciencia del comportamiento relevantes para la comprensión de la salud y enfermedad "fisica" y su aplicación al diagnóstico prevención, tratamiento y rehabilitación de la misma.

El dualismo cartesiano, vigente todavía tanto en los modelos teóricos de la medicina como en la estructura sanitaria, empieza así a ser cuestionado y, aunque raro aún por su escasa frecuencia, no sorprende ya ver a un psicólogo como integrante de un equipo de Hematología, Alergia o Cardiologia.

Hoy, gracias al desarrollo de la investigación psicofisiológica, ya nadie pone en duda que las influencias psicológicas actúan como mecanismos agravantes o desencadenantes cuando no, en muchos casos, como factores etiológicos muy delimitados. Los estilos de respuesta con que el individuo maneja su enfermedad y la experiencia psicológica del dolor junto a efectos varios de los métodos de tratamiento y el comportamiento, están identificados como aspectos que influyen o alteran el mismo proceso de la enfermedad, los resultados del tratamiento o causan otros problemas psicofisiológicos especificos.

El objetivo de nuestro trabajo es, precisamente, el de analizar algunos de estos aspectos en un trastorno psicofisiológico bien caracterizado: el asma bronquial, de interés no reciente para el autor (Bartolomé, Carrobles, Costa, Del Ser, 1976).

\section{CARACTERIZACION}

El asma bronquial es un cuadro clínico producido por una reactividad elevada del árbol traqueobronquial y bronquiolos periféricos a numerosos estímulos, caracterizado por episodios de disnea y sibilancias que son sintomáticos de la obstrucción de vías aéreas de mediano y gran calibre. En algunos pacientes puede aparecer tos con o sin producción de esputo persistente. Estos sintomas son el resultado del broncoespasmo, edema en las paredes bronquiales e hipersecreción de las glándulas mucosas. Los episodios asmáticos pueden ser continuos o paroxísticos y ocasionan una disminución variable de la función respiratoria, alcanzando desde un grado moderado de incapacidad a un estado de asfixia con amenaza de la vida (status asmático). Sin embargo, el asma es reversible, espontáneamente o a través del tratamiento (Weiss, 1978).

Además de los síntomas señalados más arriba, existen otros que ayudan o permiten caracterizar y delimitar el asma bronquial de un modo más completo. Kinsman y otros han llevado a cabo una serie de estudios (Kinsman, Luparello, O'Banion, Spector, 1973; Kinsman, O'Banion, Resnikoff, Luparello, Spector, 1974; Kinsman, Dahlem, Spector, Shucard, Luparello, 1974; Kinsman, Dahlem, Spector, Staudenmayer, 1977) en los que consiguieron aislar diez categorias de síntomas que fueron referidos por los pacientes como caracteristicos de los ataques asmáticos. Estas categorías son: pánico-miedo, fatiga, irritabilidad, hiperventilación-hipocapnia, obstrucción de vias aéreas-disnea, broncoconstricción-congestión, soledad, preocupación, cólera y respiración rápida. En estos estudios, Kinsman y otros encontraron que ciertos patrones de sintomatología parecen estar relacionados con los estilos de respuesta con que el paciente maneja su enfermedad y que influyen en los resultados del tratamiento y las decisiones médicas.

En orden a la categorización del asma 
se ha sugerido diferentes agrupamientos - clasificaciones, según una dimensión de severidad como la de Eggleston (1970): ataques esporádicos, asma suave continua, episodios frecuentes y asma severa crónica; y Freedman y Pelletier (1970): suave, persistente o severa y asma explosiva; o basada en factores etiológicos conocidos: asma intrínseca asociada con infección respiratoria, asma extrinseca producida por la reacción alérgica del paciente a agentes externos tales como el polvo o pelos de animales, y asma mixta con los dos componentes. Si bien estas últimas clasificaciones, basadas en una combinación de factores etiológi$\cos$ y variaciones clínicas conocidas, no son enteramente satisfactorias, tienen la ventaja de ayudar al clínico a una selección apropiada de la terapia para el manejo del paciente, o a establecer un factor pronóstico. Por ejemplo, el asma bronquial con procesos infectivos respiratorios puede tener un pronóstico general más pobre por la tendencia a cronificarse con el desarrollo de la tos y la producción de esputos en los intervalos intercríticos.

\section{ASPECTOS ETIOLOGICOS}

A causa de la diversidad de estímulos conocidos como productores o desencadenantes del asma, es dificil encontrar una teoria que explique satisfactoriamente todos los tipos y causas. Weiss llega a decir que con toda probabilidad el cuadro clínico de broncoespasmo, disnea, sibilancias y alteraciones respiratorias, conocido como asma, puede ser, en términos etiológicos, varias enfermedades. Se han postulado diferentes hipótesis para explicar la hipersensibilidad de las vías aéreas, característica fundamental del asma. Los alergólogos creen que este estado resulta de la respuesta anormal de la mucosa respiratoria, que permite la formación de anticuerpos a otras sustancias inofensivas tales como pólenes, pelos de animales, etc. La interacción subsecuente entre los alérgenos y los anti- cuerpos ocasiona la liberación de una gran cantidad de mediadores quimicos (histamina, serotonina, acetilcolina...) que provoca broncoconstricción, hinchazón de las células mucosas e incremento de secreción. Otras hipótesis hacen hincapié en la influencia de los receptores betaadrenérgicos y actividad parasimpática. Existe, por otra parte, un extenso número de estímulos que no causan ningún tipo de reacción alérgica y que, sin embargo, producen una alta reactividad en las vias aéreas. Este tipo de hipersensibilidad que causa una proporción significativa de ataque asmático parece ser adquirido, como veremos más adelante, por condicionamiento o bajo la influencia de factores psicológicos específicos. En cualquier caso, existe acuerdo acerca de la conceptualización del asma como una enfermedad causada por múltiples factores (ver fig. n. ${ }^{\circ} 1$ ).

Los efectos patogénicos de la hipersensibilidad en el calibre de las vías aéreas pueden verse en la figura $n .^{\circ} 2$. El tono fisiológico de la musculatura lisa de las vías aéreas bronquiales es balanceada por influencias vagales y simpáticas. La estimulación vagal causa contracción de la musculatura lisa y constricción de vías aéreas, mientras la estimulación simpática produce los efectos opuestos.

Otros factores tales como exudados purulentos, edema y fibrosis de las paredes de las vías aéreas configuran el patrón típicamente obstructivo del asma, en el que el volumen inspirado y espirado con cada ciclo respiratorio está reducido. En oposición a este proceso obstructivo existe un número de factores que ayudan a mantener el ancho de las vías aéreas (ver figura $n .{ }^{\circ} 2$ ).

La obstrucción de vías aéreas también deteriora la distribución de gases hacia el alveolo y la ventilación (el volumen de aire atrapado en una unidad de tiempo). Por esta razón las medidas espirométricas de flujo aéreo son especialmente utilizadas para determinar el grado de obstrucción espiratoria como medida objetiva de la gravedad de la condición asmática. En la mayor parte de las investiga- 
ciones realizadas se ha utilizado el Máximo Flujo de Espiración Forzada (PEFR) y el Volumen de Espiración Forzada en un segundo $\left(\mathrm{FEV}_{1}\right)$.

Se ha debatido mucho acerca de la importancia de los factores psicológiocos en la etiología del asma. La mayor parte de este debate se ha centrado sobre si los factores emocionales están o no están siempre implicados. A este respecto el ya clásico estudio de Rees (1964) demostró la relevancia de la conceptualización del asma como una enfermedad con múltiples causas, en donde el factor psicológico es una más. En el cuadro n. 1 se resumen los principales resultados del estudio de Rees: los factores psicológicos fueron considerados como una causa dominante en un 37 por 100 de los casos. Cuando el asma es originalmente inducida por un factor alérgico o infectivo, el stress psicológico puede precipitar los ataques.

Faulkner (1941) fue el primero en observar, usando un broncoscopio, la dilatación y estrechamiento bronquial en la respuesta a emociones positivas y negativas sugeridas en un sujeto no asmático.

Dekker y Groen (1956) midieron capacidad vital antes y después de exponer a los asmáticos a estímulos que cada uno había referido como capaces de desencadenar el asma. Seis de doce sujetos mostraron reducciones en la capacidad vital y tres desarrollaron asma.

Luparello y otros (Luparello, McFadden, Lyons, Bleecker, 1971) encontraron que el "poder de sugestión» tiene un efecto facilitador de ataques asmáticos en individuos cuyas reacciones alérgicas están relacionadas con la causa primaria de su desorden.

Clarke (1970) refirió que la cólera y el miedo hipnóticamente inducido podían reducir el $\mathrm{FEV}_{1}$ en tres asmáticos cuya enfermedad fue significativamente afecta$\mathrm{da}$ por factores emocionales. Posteriormente Smith, Colebatch y Clarke (1970) replicaron el estudio midiendo resistencia de vias aéreas. Sus dos sujetos también mostraron empeoramiento en el funcionamiento pulmonar siguiente al cólera y miedo hipnóticamente inducidos.

En otro estudio (Miklich et al., 1974) en el que se usaron transmisores de radio portátiles para registrar las emociones que ocurrían normalmente en dieciocho jóvenes asmáticos, se encontró en seis casos correlación negativa entre el arousal emocional y el correspondiente PEFR, es decir, que el arousal emocional reducía el flujo respiratorio. En el estudio de Costa (1979) también se observó en ocho pacientes asmáticos, de los quince que fueron expuestos a estímulos fóbicos idiosincráticos, una reducción significativa del PEFR.

Finalmente, en un estudio de Tal y Miklich (1976) se encontró que incidentes, recordados vívidamente, de intensa cólera y miedo redujeron el $\mathrm{FEV}_{1}$ en treinta y cinco niños y veintiocho niñas asmáticos crónicos. En cambio, la relajación incrementó el $\mathrm{FEV}_{1}$. Es decir, el mismo fenómeno activación emocionalrelajación sirve de base a la broncoconstricción y broncodilatación en el asma desencadenada emocionalmente.

Por otra parte, un número sustancial de estudios con animales (Dekker, Groen, 1956; Ottemberg, Stein, Lewis, Hamilton, 1958) así como investigaciones realizadas con humanos (Dekker, Pelser, Groen, 1957; Herxeimer, 1951) apoyan la hipótesis de que el asma es un desorden psicofisiológico aprendido de modo primario (Turnball, 1962; Yorkston, 1975) y/o secundario (Lachman, 1972; Ulman, Krasner, 1975).

Generalmente las aproximaciones del aprendizaje enfatizan uno de estos dos análisis: o bien el estímulo antecedente a través del condicionamiento clásico ha llegado a producir los ataques asmáticos, o bien el estímulo reforzante sirve, según un modelo operante, para mantener o exacerbar los ataques. Al parecer, la hipersensibilidad de un gran número de irritantes del entorno tales como aire frío, agua fría, dióxido de carbono, amoniaco, etc., parece ser adquirida a través del condicionamiento clásico, establecido durante o inmediatamente después de un 
ataque asmático que puede haber sido iniciado por una reacción alérgica o una infección. Después de alguna repetición de la asociación, estas sustancias pueden producir por sí solas un ataque asmático.

Por otra parte, otras muchas asociaciones del asma con ciertas emociones parecen ser establecidas a través del proceso de condicionamiento operante. Por ejermplo, un niño que tiene una gran necesidad de dependencia y miedo a la separación, puede aprender, según un paradigma operante, que los ataques asmáticos pueden llevarle más cerca de su madre y aliviar su miedo. Posteriormente estos mismos sentimientos, activados por el entorno, pueden por sí mismos desencadenar el broncoespasmo. Así pues, la sobreprotección y la atención y actitud familiar a ciertos patrones de respuesta respiratorias pueden constituir agentes causales o de mantenimiento de la respuesta asmática. Han sido varios los trabajos que han puesto de manifiesto la importancia de las relaciones familiares en la etiologia del asma (Rees, 1963; Purcell et al., 1969). Según el estudio de Rees, únicamente en 44 por 100 de los padres de asmáticos tenían actitudes satisfactorias frente al 82 por $100 \mathrm{del}$ grupo de control (padres de niños no asmáticos). En el trabajo de Purcell (1969) en el que se estudió a 22 niños asmáticos por un amplio periodo de tiempo se observó una mejoría significativa en las medidas del asma cuando los niños fueron separados de sus padres (ver fig. n. ${ }^{\circ}$ 3).

\section{PATRONES CONDUCTUALES DEL PACIEN'TE EN EL MANEJO DE SU ENFERMEDAD}

Generalmente las decisiones que se toman en el tratamiento de pacientes asmáticos se han venido haciendo en base a medidas y pruebas objetivas tales como las pruebas de sensibilidad de la piel, inhalación de antígenos (Spector, Farr, 1974), pruebas para la hiperreactividad de vías aéreas (Spector, Farr, 1975), etc.
Aun cuando todas estas evaluaciones son válidas y necesarias, existe otro tipo de medidas (sintomatología subjetiva referida por el paciente, actitudes y modos de enfrentarse a la enfermedad...) que influyen significativamente no sólo en las decisiones médicas sino también, de modo directo, en el proceso de la enfermedad. Qué duda cabe que los médicos pueden estar inclinados a tratar a los pacientes asustadizos más intensivamente (puesto que aparentan estar peor) que a los que muestran patrones conductuales más equilibrados. Es una observación común y muy generalizada que la gente reacciona diferentemente a stressores similares. En el asma la obstrucción intermitente de vías aéreas es el principal stressor y fuente de molestia común a todos los pacientes. Unos reaccionan demandando más medicación o utilizando masivamente el aerosol (broncodilatador), otros, los menos, en la tendencia a enfrentarse por sí mismos al control del broncoespasmo.

En un esfuerzo por comprender cómo la enfermedad es experimentada por los pacientes y cómo esta experiencia es traducida en la conducta que afecta el curso de la enfermedad, Kinsman y otros (Kinsman, Luparello, O'Banion, Spector, 1973; Kinsman, O'Banion, et al.; Kinsman, Dahlem, et al., 1974; Kinsman, Dahlem et al., 1977) desarrollaron el «Asthma Symptom Checklist» (ASC). Encontraron que, en efecto, ciertos patrones de sintomatología parecen estar relacionados con los estilos de respuesta con que el paciente maneja su enfermedad y que influyen en las decisiones médicas en relación con la extensión de la hospitalización (Dirks, Kinsman, Jones, et al., 1977), intensidad de corticosteroides orales prescritos en el momento del alta (Dirks, Jones, Kinsman, 1977; Kinsman, O'Banion, Resnikoff, 1973; Kinsman, Spector, Shucard, Luparello, 1974; Kinsman, Dahlem, Spector, 1977), y tasas de rehospitalización después del tratamiento residencial (Dirks, Kinsman, Horton, 1978).

La naturaleza del régimen de esteroides recomendado ha sido utilizado en 
muchos estudios como un indice de la intratabilidad del asma, debido a la filosofia que generalmente se sigue en cuanto a la administración de los mismos. Los esteroides están entre las drogas más efectivas recomendadas para el tratamiento del asma. Sin embargo representa una categoría de medicación que tiene graves efectos secundarios y, por esta razón, existen ciertas pautas de aplicación: si el asma puede ser controlada sin esteroides no se recomiendan al dar de alta al paciente; por el contrario, si el asma no puede ser manejada sin esteroides, éstos se recomiendan con un régimen de día alterno. Unicamente, si existe gravedad, es decir, si existe una gran dificultad en el control del asma, estaría indicado un régimen diario de esteroides.

En el estudio de Kinsman et al. (1977) se encontró que la frecuente ocurrencia de síntomas de "pánico-miedo» (P-M) y de "Irritabilidad" (I) del ASC, durante los ataques de asma, tiene relación con el régimen de esteroides. Los pacientes que puntuaban bajo en el factor P-M recibían regímenes menos intensivos que los altos $(\mathrm{P}<0.025)$ y los moderados $(\mathrm{P}<$. 0.05), fueron dados de alta sin esteroides más a menudo que los moderados ( $\mathrm{P}<$ 0.001) o altos $(\mathrm{P}<0.001)$ e igualmente fueron menos a menudo dados de alta sobre un régimen diario de esteroides que los moderados $(\mathrm{P}<0.001)$ y altos $(\mathrm{P}<0.05)$. Un análisis adicional mostró que estos puntajes en el factor P-M eran independientes de la frecuencia y severidad de los episodios agudos de broncoconstricción, por lo que se sugirió que los síntomas del factor P-M pueden estar asociados con conductas que alteran el cuadro clínico, llevando a los médicos a prescribir regímenes intensivos de droga a los pacientes más asustadizos. Esta posibilidad ha sido fuertemente considerada, sobre todo por la escasísima intercorrelación entre los puntajes del factor P$\mathrm{M}$ del ASC y la media del $\mathrm{FEV}_{1} / \mathrm{FVC}$ por 100 (medida de flujo respiratorio que se utiliza como indice de la broncoconstricción).

Dahlem, Kinsman, Harton (1977) en- contraron que medicaciones suplementarias, como el uso de broncodilatadores aerosolizados para aliviar la broncoconstricción, son tomados a la discreción del paciente en función de los puntajes que obtenían en el factor P-M. Pacientes con moderado puntaje en el factor P-M se inclinaban por demandas de estas medicaciones en relación a su nivel objetivo de broncoconstricción, mientras que los pacientes con alto puntaje en el factor P$M$ demandaban muchas y con bajo puntaje, pocas, con independencia del nivel de broncoconstricción. Se ha sugerido que muertes repentinas e inesperadas entre pacientes asmáticos pueden resultar de la tendencia, en pacientes con puntuaciones extremadamente bajas en el factor P-M, a minimizar o desatender sus síntomas, o en pacientes con alto puntaje en dicho factor, a sobreutilizar aerosoles de acción simpaticomimética. Dahlem y Kinsman (1978) diseñaron una investigación con el objetivo de determinar si las diferencias en el uso de medicaciones suplementarias, por los pacientes que varian en el factor P-M, resulta de las diferencias en la percepción de su estado respiratorio o de diferencias en las respuestas a percepciones similares. Los resultados sugieren que las diferencias observadas en la frecuencia de demanda de medicación entre los grupos del factor P-M no están relacionadas con las variaciones en la tasa de dificultad respiratoria. Este estudio ejemplifica una situación en donde el informe subjetivo (dificultad respiratoria) y la conducta (demandas de medicación) diverge de modo importante. Es probable que el factor P. $M$ influya en los resultados médicos del asma a causa de estrategias conductuales maladaptativas que no dependen de las diferencias en la habilidad de los pacientes en percibir y evaluar sus dificultades respiratorias.

En un esfuerzo por extender la aplicabilidad del factor "pánico-miedo» (P-M) a otras enfermedades crónicas, Dirks (1976) exploró la relación entre el factor P-M y escalas estándar del MMPI. Ninguna de éstas predijo adecuadamente los 


\section{Estudios}

puntajes de dicho factor. En línea con este trabajo, Dirks, Nelson, Jones y Kinsman (1977) diseñaron un estudio para derivar una nueva escala del MMPI, por identificación de ítems individuales relacionados con los puntajes del factor P-M. La nueva escala de personalidad P$M$ del MMPI predijo consistentemente los niveles del factor P-M del ASC en la replicación. Los resultados indicaron que los pacientes que puntuaban alto en la escala P-M del MMPI tienen, más a menudo, asignados elevados regímenes de esteroides en días alternos o diariamente, y menos a menudo prescripciones sin esteroides en el momento del alta $(P<$ 0.001). La escala P-M del MMPI predijo regimenes de esteroides igual o mayor que los puntajes del factor P-M del ASC. Sin embargo, las dos medidas no son reflejo redundante del mismo tipo de ansiedad. Usando categorías estandarizadas de Bajo, Moderado y Alto, únicamente la mitad de los pacientes asignados a estas categorias en la escala de personalidad P-M han correspondido con las asignaciones hechas en la escala de sintomatología del factor P-M del ASC. Se postula que estas diferencias reflejadas por las dos medidas se debe a que cada una de ellas indica diferentes aspectos de los estilos de respuesta con que reacciona el paciente ante el asma. La escala de personalidad P-M parecería predecir cualidades en la respuesta a dichas dificultades, alcanzando desde contradependencia (Baja personalidad P-M) a dependencia (Alta personalidad P-M). Por esta razón, los estilos de respuesta reflejados por esta escala pueden ser maladaptativos como lo indican las tasas altas de rehospitalización en tratamientos a largo plazo. En cambio, los estilos de respuesta asociados con la sintomatología del factor P-M del ASC no son probablemente maladaptativos en el asma, y pueden indicar el nivel de vigilancia de los pacientes acerca de sus dificultades respiratorias. Pacientes con alto puntaje en el factor P-M del ASC están constantemente vigilantes acerca de sus síntomas y por tanto, es probable que prevengan que las dificultades de respiración progresen hasta llegar a un ataque asmático. Por otra parte, la desatención, característico de pacientes con bajo puntaje en el factor $\mathrm{P}-\mathrm{M}$, puede ocasionar fracasos en el tratamiento, y por tanto, viene a ser una respuesta maladaptativa del paciente frente a su enfermedad. Estos fracasos van desde el no seguimiento de las prescripciones médicas por minimización de los síntomas hasta una inhabilidad para discriminar cuándo se inicia o no el proceso asmático. En este sentido, no faltan estudios (Rubinfeld, Pain, 1976) que evidencian la falta de correlación en muchos pacientes entre informes verbales del asma e índices objetivos del funcionamiento pulmonar. Es decir, una persona puede informar que está experimentando los síntomas del asma cuando las medidas objetivas indican que está libre de síntomas y, por el contrario, otra persona puede no llegar a sentir la presencia de obstrucción marcada de vías aéreas cuando las medidas objetivas de su funcionamiento pulmonar así lo indican. Bajo estas circunstancias no es de extranar que el entrenamiento en discriminación de sintomas sea obviamente necesaria en el programa de tratamiento y en especial, para aquel tipo de pacientes con una tendencia a desarrollar períodos de pequeña latencia entre el principio del asma y el status asmático (Creer, Burns, 1979).

En un trabajo reciente, Staudenmayer y otros (1979), trataron de comprender cómo la hiperreactividad de las vías aéreas (indicado por el efecto de inhalaciones de histamina y metilcolina) y/o estilos de manejo de la enfermedad (indicado por la sintomatología del factor P-M) pueden potencialmente influir los resultados médicos en pacientes asmáticos dados de alta en un medio hospitalario. Los resultados indicaron que si bien puede hacerse alguna predicción en base a los niveles de ansiedad (P-M) e hiperreactividad de vías aéreas por sí solas, la mejor predicción resultó de los efectos combinados de estos factores. Casi la mitad de los pacientes que tenían alta hiperreactividad (sobre una muestra de 196 
sujetos) y tendencia a desatender los síntomas de dificultad respiratoria fueron rehospitalizados. El nivel de atención dirigido a las dificultades respiratorias venía dado por el puntaje alcanzado en el factor P-M, oscilando entre desatención (bajo P-M) y extrema vigilancia (alto P$\mathrm{M})$. Se encontró que Bajo puntaje en $\mathrm{P}$ $M$ tiene el doble de probabilidad de ser hospitalizados (36,7 por 100) después del tratamiento que los pacientes con Alto P-M (17,5 por 100).

Si bien no se han llegado a resultados concluyentes, estos trabajos sugieren la existencia de dos tipos de ansiedad (una adaptativa y maladaptativa la otra) y plantean interrogantes a aquellos tratamientos que se basan fundamentalmente en reducir o eliminar la ansiedad de modo indiscriminado. En cualquier caso, existen aún lagunas que conviene cubrir, ya que es extremadamente dificil separar nivel de vigilancia sobre el propio síntoma del nivel de ansiedad medida por cualquier método que se utilice. Quizá sería útil el abordar los parámetros psicofisiológicos como medida objetiva de la ansiedad en uno y otro tipo de pacientes. Es posible que este tipo de investigaciones arrojen luz sobre esta problemática y contribuyan a delimitar más objetivamente los «coping styles» del paciente.

\section{APROXIMACIONES TERAPEUTICAS}

En cualquier programa terapéutico es necesario evaluar todas las posibles causas y factores que contribuyen a desencadenar las crisis asmáticas. De lo visto hasta aquí se deduce que el asma bronquial es un cuadro clínico en donde es evidente la insuficiencia de utilizar un único nivel de análisis y estrategia terapéutica. No son pocos los casos de cronificación de pacientes asmáticos con efectos irreversibles (bronquitis) posiblemente por no utilizar esos otros niveles. El modelo médico con exclusividad se ve incapaz de responder satisfactoria- mente a esa gran cantidad de problemas que se plantean cuando un paciente no responde al tratamiento habitual o cuando presenta exacerbaciones inexplicables.

Esta separación de niveles puede que posibilite en la práctica, ciertas situaciones contradictorias. Por ejemplo, en el caso de pacientes asmáticos con reacción alérgica, una de las pautas médicas a seguir con cierta frecuencia es, por una parte, la de evitar el contacto directo con aquellos estímulos (alérgenos: polvo, polen...) que pueden desencadenar la reacción asmática. $Y$ por otra parte, la administración de vacunas con estractos de antígenos o alérgenos diluidos, inyectados en dosis crecientes según un programa de hiposensibilización. Según esto, lo que en realidad se puede estar haciendo es favorecer la sensibilización del paciente a nivel conductual por medio de las prescripciones del tipo «evitar los alérgenos" cuando a nivel bioquímico se está desensibilizando al paciente mediante los programas de vacunas hiposensibilizantes. La sensibilización puede facilitarse ante la dificultad de evitar completamente la exposición del paciente a los alérgenos. De hecho, existen, inevitablemente, exposiciones parciales u ocasionales con efectos aversivos evidentes producidos por el broncoespasmo. Esto ocasiona que el paciente utilice el nebulizador y/o escape del alérgeno como medio eficaz de terminar con dichos efectos aversivos. Se produce así una reducción de ansiedad y alivio, efecto de la broncodilatación, contingente a la conducta de escape y/o evitación y uso del nebulizador. El paciente no tiene ocasión de exponerse de modo prolongado al estímulo que desencadena reacciones alérgicas y poder asi desarrollar una cierta habituación conductual al mismo.

De cualquier modo, ésta es una hipótesis que el autor de este trabajo plantea como objeto de futuras investigaciones, y fundada no sólo en la experiencia clinica y en la efectividad de tratamientos en base a la desensibilización sistemática, sino también en la existencia de trabajos experimentales controlados. En uno de 
éstos (Herxheimer, Prior, 1952) se vio que un progresivo aumento del tiempo de inhalación de alérgenos en aerosol, a través de un circuito respiratorio, tenía como resultado un aumento significativo en la tolerancia a los mismos y una relativa ausencia de crisis en pacientes asmáticos.

Planteadas estas consideraciones introductorias sobre la necesidad de abordar el problema desde una perspectiva global y no exclusivamente médica, veamos algunos aspectos o aproximaciones concretas de las estrategias terapéuticas.

Con excepción del ataque agudo y episodios que pueden progresar a un status asmático con amenaza de la vida, la mayor parte de los problemas asmáticos pueden tratarse sin requerir hospitalización. Tanto en el ataque agudo como en el status asmático, que es una situación de emergencia con alteraciones respiratorias refractarias a la terapia convencional para el episodio agudo, es necesaria la hospitalización (Weiss, 1978).

Una vez resuelto el episodio agudo o status asmático, los objetivos del tratamiento a largo plazo en cualquier paciente con asma debe ser el de prevenir tanto la ocurrencia de sintomas asmáticos como desarrollo del trastorno. Como la etiopatogenia del asma es multifactorial, del mismo modo debe recurrirse a diferentes aproximaciones terapéuticas en pro de prevenir y controlar los síntomas. El asma es un cuadro clínico en donde es evidente la interdisciplinariedad en su evaluación y tratamiento, si bien la relevancia o prepotencia de unos niveles de análisis (médico, psicológico...) sobre otros dependerá del caso concreto.

Las diferentes aproximaciones terapéuticas pueden ser agrupadas como sigue:

\subsection{Medidas generales (Weiss, 1978)}

Estas medidas incluyen una alimentación con dieta no alérgica, descanso y sueño adecuado y una razonable cantidad de actividad y ejercicio físico. Por otra parte, el paciente debe evitar ciertos estímulos que pueden ser desencadenantes de crisis asmáticas tale como la fatiga, humedad, inhalación de sustancias químicas volátiles, humo de tabaco, temperaturas extremas, contacto con individuos con infección respiratoria, polen y otros alérgenos. El uso de humidificadores es importante durante el verano, cuando un descenso de la humedad puede causar irritación a las membranas mucosas, y la sinusitis y trastornos de la garganta deben ser tratados con cierta urgencia, ya que pueden ser desencadenantes de episodios asmáticos.

\subsection{Medicación (Weiss, 1978)}

Existen medicaciones que tienen el poder de hacer reversible el broncoespasmo como las catecolaminas (epinefrina) que actúan sobre los receptores Badrenérgicos y agentes que inhiben la enzima fosfodiestarasa (aminofilina) facilitando el aumento de la capacidad vital y la reducción de la resistencia de vías aéreas. Estos últimos están indicados preferentemente en hipertensivos, hipertiroideos y pacientes cardiacos dado que los efectos secundarios cardiovasculares son menores que con las catecolaminas. También se utilizan los corticosteroides como resultado de su múltiple acción sobre los receptores B-adrenérgicos y la enzima fosfodiestarasa, sin embargo, tienen el inconveniente de sus múltiples efectos secundarios, de rara aparición en terapias de corta duración. En un tratamiento crónico o más o menos largo, la administración de corticosteroides puede causar supresión suprarrenal, edema, hipertensión, agravación de la diabetes mellitus, exacerbación o extensión de la infección, miopatía, ulceración péptica con hemorragia... Por todos estos efectos secundarios, y aunque pueden utilizarse otros agentes (sodio, potasio...) que los limitan o previenen, es aconsejable el uso de esteroides cuando fallan otras terapias farmacológicas, en casos graves, y siempre con precauciones en cuanto a las dosis y regímenes. 


\subsection{Hiposensibilización (Weiss, 1978)}

Un tratamiento de hiposensibilización está especialmente indicado para pacientes con asma extrínseca o mixta en que el factor alérgico juega un importante papel. En este tratamiento se administran extractos de antígenos diluidos en dosis gradualmente crecientes hasta alcanzar un máximo de adaptación del organismo y eliminar de este modo la reacción alérgica.

\subsection{Manejo comportamental}

Esta es el área específica del nivel de análisis e intervención del psicólogo.

\subsubsection{Análisis conductual}

Además de la utilización de medidas objetivas (PEFR, FEV 1 , TRR) y colaterales (duración de la tos, número de ataques, cantidad de medicación, síntomas subjetivos...) de la condición asmática, la intervención comportamental debe ir siempre precedida de un análisis funcional riguroso de la conducta asmática y factores del entorno. Estos pueden ser resumidos y categorizados en:

- Estimulos antecedentes. asma.

- Estímulos que concurren con el

- Estímulos consecuentes.

\subsubsection{Estimulos antecedentes. Estos es-} tímulos se definen como aquellas conductas (en sus dimensiones motoras, fisiológicas y cognitivas) o circunstancias que ocurren antes del comienzo de un ataque asmático. Suelen tener la virtualidad de facilitar o desencadenar la respuesta asmática por lo que resulta de importancia crucial su detección y control. En un reciente trabajo (Costa, 1979) quince pacientes asmáticos seleccionados aleatoriamente registraron, durante dos meses, las condiciones antecedentes de sus crisis asmáticas. Estas variaron desde la exposición del paciente a estímulos con poder de desencadenar respuestas emocionales, hasta conductas de implicación en ejercicio físico y padecimiento de catarros (ver tabla $n .{ }^{\circ} 2$ ).

En otro estudio, Eney y Goldstein (1976) encontraron que de un grupo de 43 pacientes seleccionados al azar, el 88 por 100 no tomaba sus medicaciones tal y como se les había prescrito. El no seguimiento de la medicación viene a ser otra condición antecedente algo generalizada en pacientes asmáticos.

Por todas estas razones toda evaluación de "estímulos antecedentes» debe comprender el estudio de:

\section{- Seguimiento o no de la medicación.}

- Exposición a estímulos específicos: polvo, plumas, gasolina, pinturas, plantas, humos, aire frío, etc.

- Exposición a estímulos con poder de desencadenar respuestas emocionales. Estímulos fóbicos: mercado, metro, desorden, "percatarse» de no llevar el nebulizador, haber discutido con..., estar pensando en...

- Implicación en conductas de comer, inyectarse, ingesta de medicamentos, hacer ejercicio, etc.

- Enfermedades: catarros, etc.

4.4.1.2. Estimulos concurrentes. Las condiciones que concurren con un ataque asmático pueden ejercer un efecto paliativo o de exacerbación del mismo. Ya se vio como los patrones conductuales con que reacciona el paciente o la capacidad de discriminar en qué momento se inician los síntomas asmáticos tienen una importancia crucial en el desarrollo de la condición asmática. Así, pues, la evaluación de "estímulos concurrentes» debe comprender:

- Características sintomáticas: pánicomiedo, fatiga, irritabilidad, congestión mucosa, tos, opresión torácica, calambres, mareos, etc.

- Discriminación sintomática: discrepancia entre los informes de sentimientos subjetivos e indices objetivos.

- Otras conductas implicadas en el mane- 
TABLA 2.

Estímulos antecedentes a las crisis asmáticas (M. Costa, 1979).

\begin{tabular}{|c|c|c|c|c|c|c|c|c|c|c|c|c|c|c|c|c|}
\hline Sujetos asmáticos & 1 & 2 & 3 & 4 & 5 & 6 & 7 & 8 & 9 & 10 & 11 & 12 & 13 & 14 & 15 & \\
\hline Sexo & $V$ & $H$ & $H$ & $H$ & $H$ & $H$ & $V$ & $H$ & $H$ & $H$ & $H$ & $H$ & $H$ & $H$ & $H$ & \\
\hline \multicolumn{17}{|l|}{$\begin{array}{l}\text { Estimulos precedentes a } \\
\text { la crisis: }\end{array}$} \\
\hline Discusión & 6 & & & 4 & & & 4 & & & & & 3 & & & & 17 \\
\hline Polvo doméstico & 8 & 9 & & 6 & 2 & & & 4 & & & 3 & & 4 & & & 36 \\
\hline $\begin{array}{l}\text { Droguería } \\
\text { «Percatarse» de no }\end{array}$ & 2 & & & & & & & & & & & & & & & 2 \\
\hline llevar aerosol & 2 & 1 & 3 & & & & & & & & & & & & & 6 \\
\hline $\begin{array}{l}\text { Pensar en ataque } \\
\text { asmático } \\
\text { Tareas domésticas }\end{array}$ & & & 8 & & & & & & & & & & & & & 8 \\
\hline $\begin{array}{l}\text { Tareas domésticas } \\
\text { (desor.) } \\
\text { Conduct. Problema }\end{array}$ & & 6 & 10 & 3 & & 10 & & & 2 & 6 & 11 & & 3 & 3 & & 54 \\
\hline familiar & & & & 4 & & 8 & & & 2 & & & 5 & & & & 19 \\
\hline $\begin{array}{l}\text { Lugares «cargados» } \\
\text { y cerrados }\end{array}$ & & & & & & & & & & & & & & & & \\
\hline $\begin{array}{l}\text { y cerrados } \\
\text { Contacto con lana }\end{array}$ & & & & 3 & & & & & 2 & & & & & & & 5 \\
\hline $\begin{array}{l}\text { Contacto con lana } \\
\text { Aglomeraciones }\end{array}$ & & & & & 2 & 7 & & & & & & & & & & 2 \\
\hline $\begin{array}{l}\text { Aglomeraciones } \\
\text { Salir a la calle }\end{array}$ & & & & & & t & & 2 & & & & & & & & 9 \\
\hline $\begin{array}{l}\text { Salir a la calle } \\
\text { Percibir sensación de }\end{array}$ & & & & & & & & & & & & & & & & 8 \\
\hline «inestabilidad» & & & & & & & & 6 & & & & & & & & 6 \\
\hline Esfuerzo físico & & & 5 & & & & & & & & & & & & & 5 \\
\hline Catarros & & 4 & & & & 5 & & & & 4 & 4 & 2 & 1 & & 1 & 21 \\
\hline Inhalación de polen & & & & & & 6 & & 4 & & & & & & & & 10 \\
\hline Sin determinar & & 4 & 4 & & & & & & 2 & 5 & & 2 & 2 & 3 & 5 & 27 \\
\hline$N^{\circ}{ }^{\circ}$ total de crisis & 18 & 24 & 30 & 20 & 4 & 36 & 4 & 24 & 8 & 15 & 18 & 12 & 10 & 6 & 6 & 235 \\
\hline
\end{tabular}

jo del asma: utilización del aerosol, utilización o no de habilidades de relajación, llamadas urgentes a familiares...

4.4.1.3. Estimulos consecuentes. $\mathrm{Ya}$ se vio en otra parte de este trabajo la relevancia del componente operante en la causación y mantenimiento de la respuesta asmática. Por esta razón, es una condición necesaria de toda evaluación el análisis de sus consecuencias.

- Atención familiar.

- Prolongación de la bospitalización.

- Evitación de situaciones aversivas: ir a la escuela...

- Reducción de disconfor: interrupción de discusiones...
(Ver tabla n. ${ }^{\circ}$ ).

Dado que los factores que mantienen una enfermedad pueden ser diferentes de aquellos que la iniciaron, el análisis comportamental debe ser realizado incluso en aquellos casos de una clara etiología alérgica, infecciosa o no comportamental.

Una vez realizado el análisis funcional, la estrategia de intervención irá dirigida a modificar aquellas condiciones antecedentes, concurrentes y consecuentes que tienen un valor efectivo en el desencadenamiento y mantenimiento de la conducta asmática.

\subsubsection{Objetivos}

En un sentido amplio, una estrategia de intervención adecuada debe compren- 
TABla 3.

-Enfermedades: catarros.

- Fobias: aglomeraciones.

-Est. Espec.: polen, polvo.

Situacionales

-Discusión.

- Inyectarse.

- Comer.

- Ingerir el medicamento.

- No seguir la medicación.

-Esfuerzo fisico.

- "No llevo el nebulizador" - "Me va a dar una crisis."

-"Qué horror!m

- "Si respiro polvo me da.»

Estimulos

Antecedentes

Cognitivos

Cosiro polvo me da

- Colapso dinámico durante la exp. forzada o tos.

-Tono del músculo liso de la pared bronquial aumentada por est. vagal o directamente en respuesta

a la tos, gases irritantes, alérge-

Quimico-

Fisiológicos

nos, mediadores quimicos.

- Hipersecreción de mucus

-Edema.

- Reacción alérgica.

- Sensaciones fisiológicas.

-Sensación de ahogo.

\begin{tabular}{l|l} 
Emocionales & $\begin{array}{l}\text {-Ansiedad } \\
\text {-Cólera. } \\
\text {-Miedo. }\end{array}$
\end{tabular}

\section{CONDUCTA}

ASMATICA

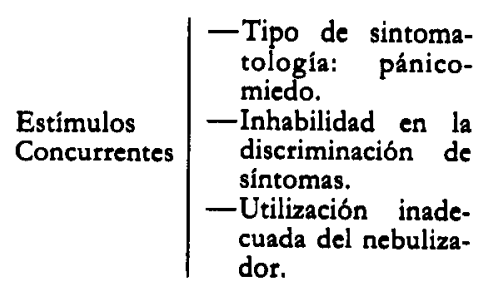

Estimulos

consecuentes

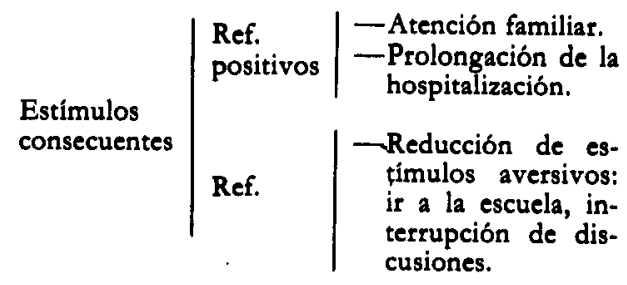


der una serie de objetivos, en los que se incidirá, en mayor o menor grado, dependiendo de la caracterización de la entidad asmática de que se trate. Estos objetivos pueden ser resumidos en:

\subsubsection{Desarrollar babilidades de observa-} ción y discriminación a fin de que el paciente detecte estímulos potenciales (cognitivos, fisiológicos y del entorno) con capacidad de desencadenar respuestas asmáticas, y pueda aprender a discriminar suaves cambios en su funcionamiento respiratorio. En los niños asmáticos es necesario también desarrollar programas detallados tendentes a una mayor autorresponsabilización en el autorregistro y automanejo de las medicaciones y del asma en general (Parcel, Nader, 1977).

\subsubsection{Desarrollar estrategias y babilida-} des en el control y reducción de la ansiedad, cólera y otras respuestas emocionales. $\mathrm{La}$ razón de este objetivo es doble: este tipo de respuestas emocionales y, en especial, la ansiedad, tienen, por una parte, un efecto broncoconstrictor, y por otra, facilitan el desarrollo de conductas maladaptativas en el manejo del asma. Recordamos aquí que pacientes que puntuaban muy alto en el factor P-M del ASC tendían a sobreutilizar inhaladores con preparaciones de aerosol. La autoutilización de estos productos y el efecto reductor de ansiedad, por el alivio o eliminación contingente de las dificultades respiratorias, puede facilitar una cierta «dependencia» en el paciente. Este comienza a sobreutilizar el inhalador de un modo compulsivo, propiciando así ciertos efectos cardiotóxicos, con peligro de muerte, como resultado de la sobredosis. Es relativamente frecuente la imagen del asmático con miedo a separarse de su nebulizador y desarrollando rituales obsesivos de comprobación. Es tal la dependencia a la que pueden llegar algunos pacientes asmáticos, que el hecho de "comprobar" que les falta el aerosol puede desencadenar respuestas de ansiedad y posteriormente ataques asmáticos. En nuestro trabajo (Costa, 1979), de una muestra de 15 pacientes asmáticos, y tras una observación de dos meses, tres de ellos tuvieron crisis asmáticas, precedidas por una comprobación de este tipo, en un 11,11 por $100,0,41$ por 100 y 10 por 100 del porcentaje total respectivo de crisis (ver tabla 2).

Un objetivo deseable adicional pues, sería el de facilitar el autocontrol del paciente de modo que desarrolle conductas adaptativas para enfrentarse por si solo a su trastorno respiratorio.

\subsubsection{Reducir la sensibilidad del pacien-} te a aquellos estímulos (polvo, polen...) que parecen tener el poder de desencadenar la respuesta asmática. Obviamente, no sólo estamos aludiendo a una reducción de la sensibilidad a nivel bioquímico mediante intervenciones directas del tipo de vacunas hiposensibilizantes, sino sobre todo a nivel conductual tanto en sus dimensiones motoras, fisiológicas como cognitivas. Sería interesante el desarrollar algún tipo de investigación tendente a determinar los efectos que pudieran tener intervenciones conductuales en el componente alérgico del asma.

\subsubsection{Control de consecuencias reforzantes} de la condición asmática, ya sean de tipo positivo (sobreprotección...) o por reforzamiento negativo (interrupción de conflictos, escape de otros estímulos aversivos...).

\subsubsection{Técnicas y procedimientos terapéuticos}

Los procedimientos de relajación y desensibilización han sido de los más utilizados y con variantes que van desde los métodos de Jacobson y Schulz hasta el de feedback EMG (Davis y cols., 1973; Scheer y cols., 1975). En la desensibilización las jerarquias utilizadas suelen realizarse con estímulos relacionados con los ataques asmáticos, con estímulos que son desencadenantes directos de los mismos y con circunstancias estressantes psicológicamente idiosincráticas de un sujeto (aglomeraciones, desorden, relaciones in- 
terpersonales...). Las exposiciones suelen hacerse de un modo muy gradual acercándose al modelo más ortodoxo de Wolpe, a fin de evitar el desencadenamiento de crisis asmática. En este sentido, convieve advertir del grave peligro que puede correr determinado tipo de pacientes por su paso rápido al status asmático.

Tanto la relajación como la desensibilización tienen un efecto significativo en la mejoría de la condición asmática (Napp, Wells, 1978) si bien parece ser que el segundo procedimiento es superior al primero (Yorkston y cols., 1974).

Se ha empleado también entrenamiento asertivo (Walton, 1960) como medio de controlar la ansiedad producida por la relación interpersonal, reestructuración cognitiva (Rathus, 1973; Costa, M. 1979), autocontrol con demora del uso del broncodilatador (Sirota, Mahoney, 1974). En un trabajo de Hock y cols. (1978) realizado con el propósito de evaluar varios modos de intervención psicológica, se encontró que el Entrenamiento en Relajación por sí solo y combinado con Entrenamiento Asertivo incrementó el funcionamiento respiratorio y redujo el número de ataques. El Entrenamiento Asertivo por sí solo fracasó en mejorar la función respiratoria y tuvo una tendencia en incrementar la frecuencia de ataques asmáticos.

El feedback de la resistencia total respiratoria (TRR) determinada por la técnica de oscilaciones forzadas parece ofrecer posibilidades en el control voluntario del tono bronquial y por tanto, en el alivio de la condición asmática. Sin embargo, los resultados hasta ahora obtenidos
(Vachon, Rich, 1976) no son muy concluyentes por la magnitud reducida del cambio y por las poblaciones elegidas. En el estudio de Vachon y Rich no existían pacientes con asma grave y necesitados de medicación del tipo corticosteroides. Estos mismos autores refieren que la magnitud del cambio referido es equivalente a la observada después de una inhalación de Isoproterenol por lo que pocos asmáticos estarían satisfechos con este nivel de alivio clínico.

Este procedimiento fue desarrollado por Dubois, Brody, Lewis y Burgess (1956), simplificado por Goldman, Knudson, Mead, Peterson, Shwaker y Wohl (1970) y automatizado por Levenson (1974). Los detalles de la técnica pueden encontrarse de modo pormenorizado en Vachon y Rick (1976) y Levenson (1974) y de modo reducido en Feldman (1976). Básicamente, este procedimiento persigue entrenar al paciente a reducir la resistencia respiratoria de las vías aéreas mediante una información (feedback) amplificada y traducida en señales acústicas o visuales acerca del nivel de resistencia de los tubos bronquiales. Según un paradigma de aprendizaje operante, el paciente aprende a modificar este nivel.

Otras técnicas operantes (time-out, response-cost, shaping, modeling...) han sido usadas efectivamente para tratar conductas asociadas con el asma (Knapp, Wells, 1979) y para desarrollar programas educativos mediante videotape (Moldojsky, Broder, Davis, Leznoff, 1979).

\section{Referencias}

Bartolomé, P., Carrobles, J. A. I., Costa, M., Del Ser, T.: La práctica de la terapia de conducta. Ed. Pablo del Rio, 1976, 2.: edición, 1979.

Ciminero, A. R., Doleys, D. M., WILliams, C. L.: Journal literature on bebavior tberapy 1970-1976: analysis of tbe subject characteristics, target bebaviors, and treatment techniques. Journal of behavior therapy and experimental psych. Volume 9, Number 4, December 1978, pp. 295-306.

CLARKE, PS.: Effects of emotion and cougb or airvays obstruction in astma.

Costa, M.: Fartores psicológicos y asma bronguial. Trabajo de investigación realizado en el Gran Hospital en abril-julio, 1979 (sin publicar).

CREER, T. L., BURNS, K. L.: Self-Management Training for Cbildren witb Cbronic Broncbial Astbma. Psychoterapy and Psychosomatics, Vol. 32, pp. 270-278 (1979). 
Dahlem, N. W., Kinsman, R. A., Horton, D. J.: Panic-Fear in astbma: requests for as-needed (PRN) medications in relation to pulmonary function measurements. Journal of Allergy, Clin. Inmunol., Pp. 60, 295-300, 1977.

Dahlem, N. W., Kinsman, R. A.: Panic-Fear in Asbtma: A Divergence Between Subjective Report and Bebavioral Patterns. Perceptual and Motor Skills, 46, 95-98, 1978.

DAvis, M. H. y otros: Relaxation training facilitated by biofeedback apparatus as a supplemental treatment in broncbial astma. Journal of Psychosomatic Research, 17, pp. 121-128, 1973.

Davison, G. C., Neale, J. M.: Abnormal psycbology and experimental clinical approarch. Ed. John Wiley and Sous. $2 .^{2}$ ed. 1978 , pp. $182-188$.

DekKer, E., Groen, J. J.: Reproducible psycbogenic attacks of astbma. L. Psycosomat. Res. 1, pp. 58-67, 1956.

Dekker, E., Pelser, H. E., Groen, J. J.: Conditioning as a cause of asthmatic attacks: $A$ laboratory study. J. Psychosom. Res. 2, pp. 97-108, 1957.

Dirks, J. F., Kinsman, R. A., Horton, D. J. y otros: Panic-Fear in astbma: Rebospitalization following intensive long-term treatment. Psychosomatic Medicine, 40, pp. 5-13, 1978.

DiRks, J. F., KInSMan, R. A., Jones, N. F. y otros: Panic-Fear: A personality dimension related to lengyb of bospitalization in respiratory illness. J. Asthma. Res. 14, pp. 61-71, 1977.

DiRKs, J. F., y otros: Panic-Fear: A personality dimension related to intractability in astbma. Psychosomatic Medicine, vol. 39, núm. 2 (March-April, 1977).

Dubois, A. B., Brody, A. W., Lewis, D. H., Burgess, B. F.: Oscillation mechanics of lung and cbest in man. Journal Appl. Physiol. 8, pp. 587-594, 1956.

ENEY, R. D., GOLDSTEIN, E. O.: Compliance of chronic asthmatics with oral administration of theophylline as measured by serum and salivary levels. Pediatrics, vol. 57, pp. 513-517, 1976.

FAULKNER, W. B.: Influence of suggestion on size of broncbial lumen. Northwest Med., 40, 367, 1941.

Feldman, G. M.: The Effect of Biofeedback Training on Respiratory Resistance of Astbmatic Cbildren. Psychosomatic Medicine, vol. 38, n. 1 , pp. 27-34, January-February, 1976.

FISCHER, A. B., DuBOIS, A. B., HYDE, R. W.: Evaluation of the forced oscillation technique for the determination of resistance to breathing. Journal Clin. Invest., 47, pp. 2045-2057, 1968.

Freedman, S. S., Pelletier, G. A.: Astbma in chilbood: Treatment of 1.070 cases. Ann. Allergy. Vol. 28, pp. 133-141, 1970.

Goldman, M. y otros: A simplified measurement of respiratory resistance by forced oscillation. Journal Appl. Physiol. 28, pp. 113-116, 1970.

HER XHEIMER, H.: Induced asthma in man. Lancet, 23 June, pp. 1337-1341, 1951.

Hock, y otros: Medico-Psychological Interventions in Male Asthmatic Children: An Evaluation of Pbysiological Change. Psychosomatic Medicine, vol. 40, n. 3 , May, 1978.

KHAN, A. V.: Effectiveness of biofeedback and conter-conditioning in the treatment of bronchial asthma. Journal of Psychosomatic Research, vol. 21, pp. 97-104, Pergamon Press, 1977.

Knapp, T. J., Wells, L. A.: Bebavior Therapy for Astbma: $A$ Reviev. Behavior Research and Therapy, vol. 16, pp. 103-115, 1978.

LaChMAN, S. J.: Psychosomatic disorders: A behavioristic interpretation. John Wiley, New York, 1972.

LEVERSON, R. W.: Automated System for Direct Meassurement and Feedback of Total Respiratory Resistance by the Forced Oscillation Tecbnique. Psychophysiology-Instrumentation, vol. 11, n. ${ }^{\circ}$, January, 1974.

Kinsman, R. A., Dahlem, N. W., SPECtor, S., Staudenmayer, H.: Observations on subjective symptomatology, coping behavior and medical decisions in asthma. Psychosomatic Medicine, vol. 39, n. ${ }^{\circ}$ 2, March-April, 1977.

KINSMAN, R. A., SpECTOR, S., y otros: Subjective symptous of acute astbma within a beterogenus sample of astbmatics. Journal of Allergy Clin. Inmunol. 52, pp. 284-296, 1973.

KInSMan, R. A., LUPARELlo, T. J., y otros: Multidimensional analysis of the subjective symptomatology of astbma. Psychosomatic Medicine, vol. 35, pp. 250-267, 1973.

Kinsman, R. A., y otros: Observations on patterns of subjective symptomatology of acute asthma. Psychosom. Med., 36, pp. 129-143, 1974.

LupAREllo, T. J., y otros: Psychologic factors and broncbial astbma. New York, State Jornal of Medicine, 71, pp. 2161-2165, 1971.

LUPARELLO, T. J., y otros: Influences of suggestion on airway reactivity in astbma subjects. Psychosomatic Medicine, vol. 30, pp. 819-825, 1968.

MikliCh, D. R., CHAI, H., PURCELl, K. y otros: Naturalistic observation of emotions preceding low pulmonary flow rates. J. Allergy Clin. Inmunol, 53, 102, 1974.

MOLDOFSKY, H., BRODER, I., DAvies, G., LEZNOFF, A.: Videotape educational program for people with asthma. CMA Journal, March 17, vol. 120, 1979.

Ottenberg, P., Stein, M., Lewis, J., Hamilton, C.: Learned asthma in the guinea pig. Psychosomatic Medicine, 20, 395-400, 1958.

PARCEL, G. S., NADER, P. R.: Evaluation of a Pilot School Healtb Education Program for Asthmatic Children. The Journal of School Health, pp. 453-456, October 1977.

PURCELL y otros: The effect on asthma in children of experimental separation from the family. Psychosomatic Medicine, 31, pp. 144-164, 1969. 
RATHUS, S. A.: Motoric, autonomic, and cognitive reciprocal inbibition of a case of bysterical bronchial astbma. Adolescence, 8, pp. 29-32, 1973.

REES, L.: The importance of psychological, allergic and infective factors in childbood. Journal of Research, 7, Pp. 253-262, 1964.

Rubinfeld, A. R., PAin, M. C. F.: Perception of asthma. Lancet, April 26, pp. 882-884, 1976.

SCHERR, M. S., y otros: Effect of bio-feedback tecbniques on chronic astbma in a summer camp enviroment. Ann. Allergery, 35, pp. 289-295, 1975.

SMITH, M. M., COLEBATCH, J. H., CLARKE, P. S.: Increase and decrease in pulmonary resistance with bipnotic suggestion in asthma. Am. Rev. Resp. Dis. 102, 236, 1970.

SPECTOR, S. L., FARR, R. S.: Broncbial inbalation procedures in astbmatics. Med. Clin. North. Am., 58, PP. $71-84,1974$.

SPECTOR, S. L., FARR, R. S.: A comparison of mentbcholine and bistamine inbalations in asthmatics. Journal of Allergy Clin. Inmunol. 56, pp. 308-316, 1975.

Sirota, A. D., MAHONEY, M. J.: Relaxing on cue: The self regulation of astbma. Jornal of Behav. Ther. and exp. Psychiat., 5, Pp. 65-66, 1974.

StAudenmayer, H., Kinsman, R. A., Jones, N. F.: Attitudes toward respiratory illness and bospitalization in asthma. The Journal of Nervous and Mental Disease. Vol. 166, n. ${ }^{\circ}$, pp. 624-634, 1978.

Staudenmayer, H. y otros: Medical Outcome in Asthmatic Patiens: Effects of Airways Tlyperreactivity and Sumptom-Focused Anxiety. Psychosomatic Medicine, vol. 41, n. 2, March, 1979.

Turnball, J. W.: Asthma conceived as a learned response. J. Psychosomatic Res. 6, pp. 59-70, 1962.

TAL, A., Miklich, D. R.: Emotionally Induced Decreases in Pulmonary Flow Rates in Astbmatic Cbildren. Psychomatic Medicine, vol. 38, 3, pp. 190-199, May-June, 1976.

Ullman, L., Krasner, L.: A psycbological approacb to abnormal bebavior. Second Edition, Engelwood Clitts N.J.

VACHON, L., RICH, E. S.: Visceral learning in Asthma. Psychosomatic Medicine, vol. 38, n. ${ }^{\circ}$, May-April, 1976.

WeIsS, E. B.: Bronchial Asthma. Medical Symposia, vol. 27, n. ${ }^{\circ}$ and 2, 1978. 